\title{
Working Conditions in the Laboratory for Raman Microscopy
}

\author{
Steva Lević
}

\begin{abstract}
Raman spectroscopy/microscopy technique allows monitoring of various chemical processes in real time, and material characterization in terms of the distribution of individual chemical components via method known as Raman mapping. Equipping laboratory for this technique requires following: a stable supply of electricity, anti-vibration table for the instrument, constant temperature and controlled source of light. Prior to beginning the measurement it is necessary to select the laser of the appropriate wavelength and to calibrate the instrument.
\end{abstract}

The instruments that are used in Raman spectroscopy/microscopy allow monitoring of various chemical processes in real time, and material characterization in terms of the distribution of individual chemical components via method known as Raman mapping. To carry out complex analyzes of sample's surface it is necessary to possess a Raman microscope with motorized stand that allows automatic scrolling and analyzing previously given points on the sample. The resultant spectra form a "chemical map" of the sample.

\section{How to cite this book chapter:}

Lević, S. 2019. Working Conditions in the Laboratory for Raman Microscopy. In:

Vucelić Radović, B., Lazić, D. and Nikšić, M. (eds.) Application of Molecular

Methods and Raman Microscopy/Spectroscopy in Agricultural Sciences and Food

Technology, Pp. 151-159. London: Ubiquity Press. DOI: https://doi.org/10.5334/

bbj.j. License: CC-BY 4.0 
In order to work in accordance with the requirements of the sample analysis, laboratory for Raman microscopy should meet several basic conditions. First of all, microscope needs a stable supply of electricity. It is necessary to eliminate sources of vibration that could adversely plug on the operation of the microscope, by placing it on the appropriate anti-vibration table (Figure 1). In addition, air-conditioning in the room is necessary in order to ensure conditions for the work of staff but also the stable temperature required for the smooth operation of the device.

In addition to the elimination of the effect of vibration, it is necessary to eliminate the influence of ambient light on the analysis in progress (Figure 2), which is achieved by shutting down the lights during excitation of the sample or by using microscope equipped with special curtain. Small table lamps that don't affect the operation of the device can be used.

\section{Selection of laser of the appropriate wavelength i.e. choice of instrument}

The first and basic condition for successful implementation of analysis by Raman microscopy is selection of the device i.e. laser. Older device types usually have only one laser that allows use of only one wavelength. Modern Raman

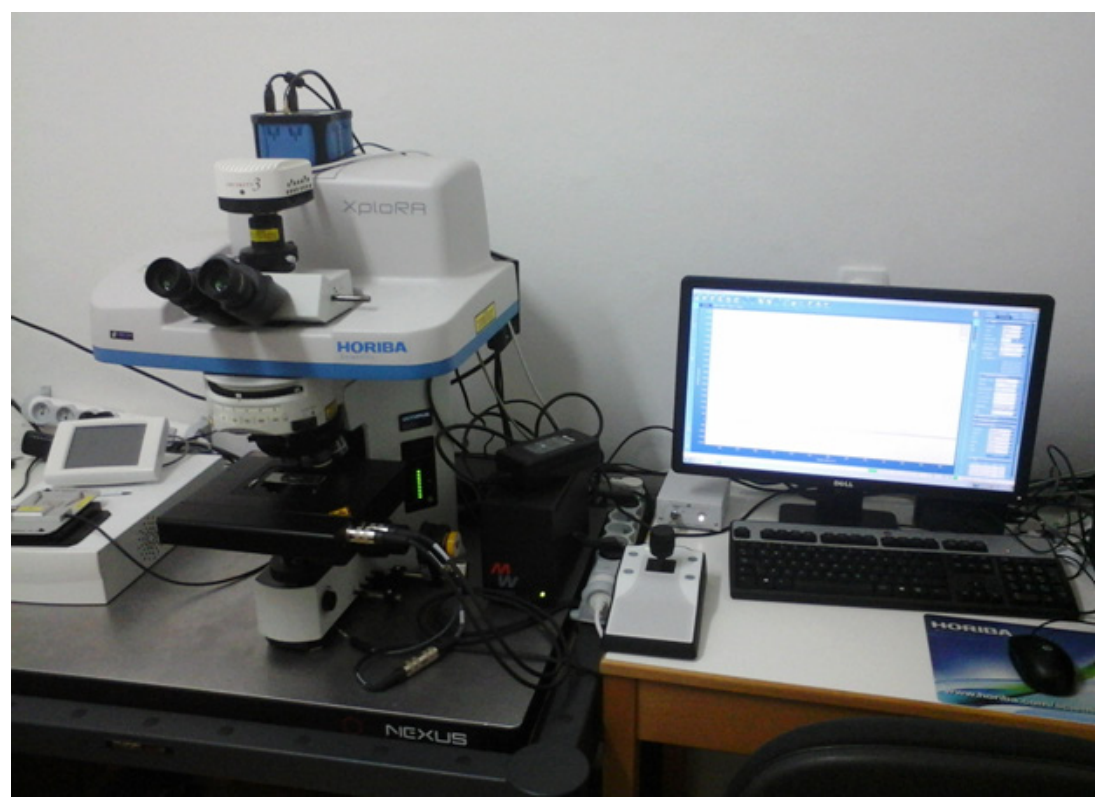

Figure 1: Raman microscope XploRA (Horiba Jobin Yvon) is set to the appropriate anti-vibration table. 


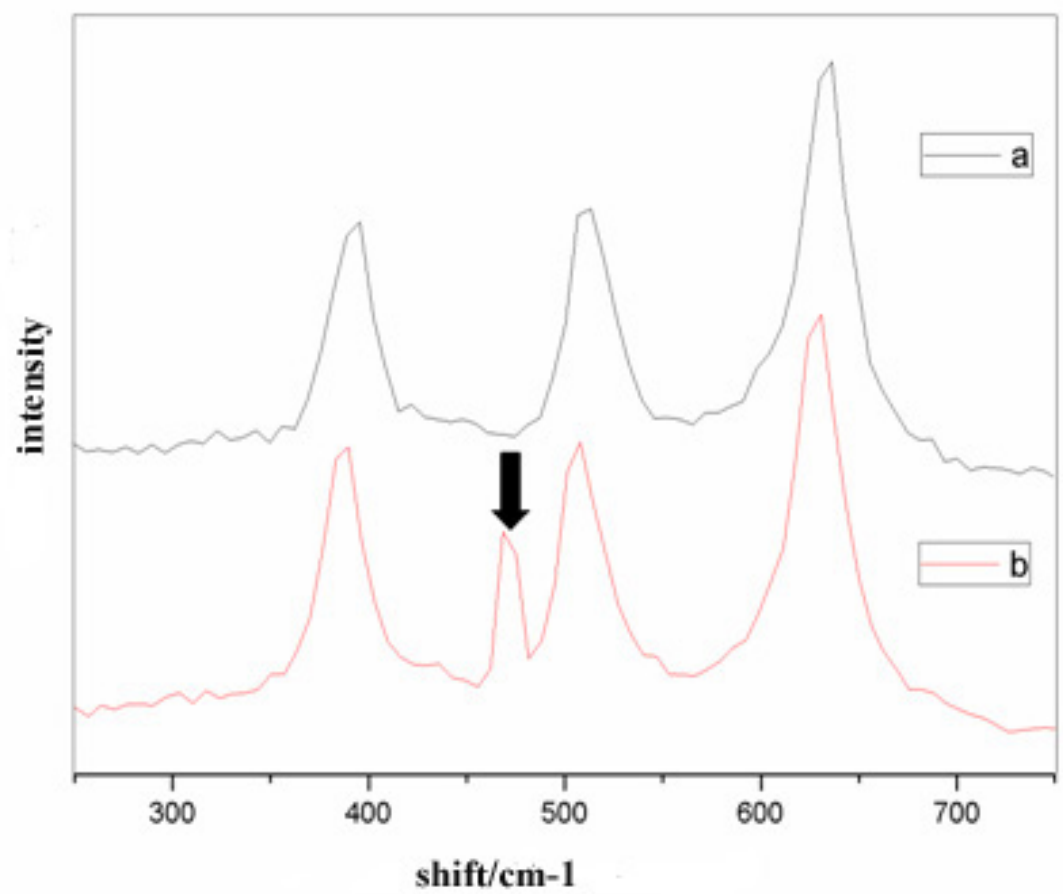

Figure 2: Influence of ambient light on the Raman spectrum of titanium dioxide. a) Recorded range without the influence of ambient light; b) includes the range of ambient light. The arrow marked signal from the ambient light. Laser $532 \mathrm{~nm}$, acquisition time of $1 \mathrm{~s}$.

microscopes can be equipped with multiple lasers allowing quick switch from one source of excitation to another depending on the needs of the analysis or properties of analyzed material.

Selecting the appropriate laser primarily depends of the nature of tested material and its interaction with the laser. Specifically, by using certain lasers (e.g., $532 \mathrm{~nm}$ ) fluorescence whose intensity exceeds the Raman signal (signal completely obscured) may occur. This phenomenon can be expressed so much that any technique for removing the fluorescence does not give any result. In such cases, lasers operating in higher wavelength range where the fluorescence is lesser or none (785 nm or $1064 \mathrm{~nm}$ ) can be selected.

\section{Calibration of the instrument}

Prior to beginning the measurement it is necessary to calibrate the instrument. This is usually done by using pure substances of known positions of 


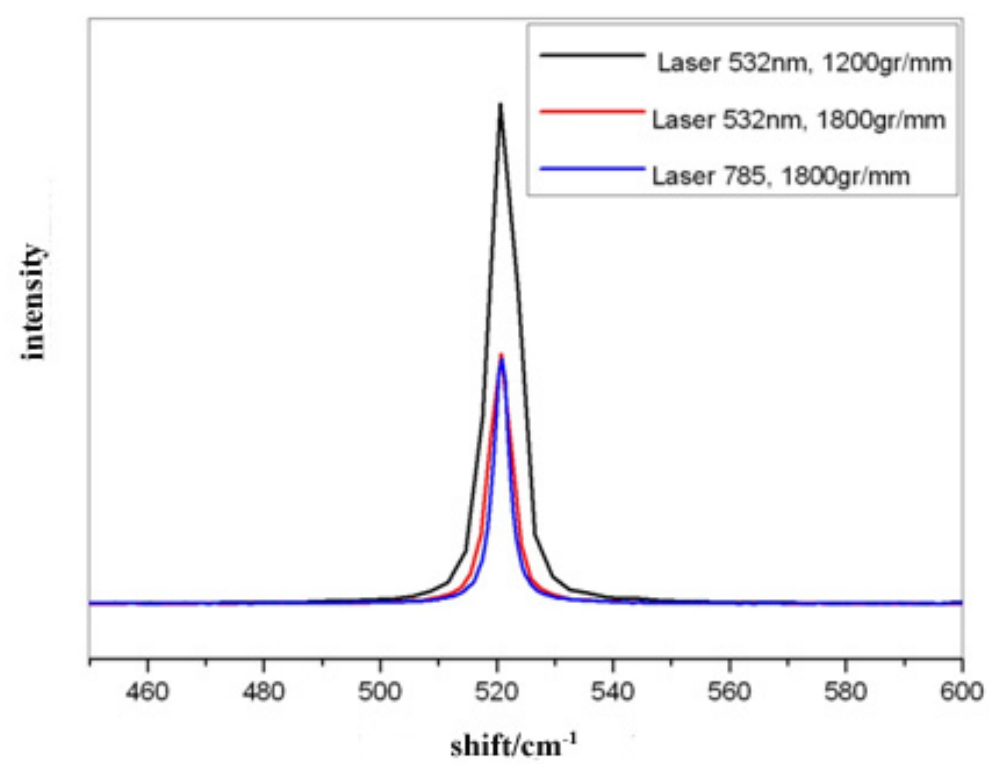

Figure 3: The result of using different lasers for calibration (calibration with silicon).

Raman signals in their spectra. Following substances can be used for calibration: silicon, sulfur, barium, etc. Example of calibration of Raman microscope XploRA (lasers at $532 \mathrm{~nm}$ and $785 \mathrm{~nm}$ ) with silicon (peak at $520 \mathrm{~cm}^{-1}$ ) is shown in Figure 3.

Procedure of calibration depends on the type of device and the calibration procedure recommended by the manufacturer. Modern Raman microscopes have adequate indicator showing whether the calibration was made in accordance with the recommended procedure.

\section{Procedure with samples}

When it comes to working on Raman microscope, one should pay attention to some basic steps that should be taken before the analysis. The first and basic step is sample preparation prior to the measurement and the method of its conservation. It should be noted that the final form of the sample must be adopted to the dimensions of the microscope and its capabilities.

Generally, for Raman microscopy glass, quartz or gold (nanometer layer on the plate) microscopy slides are used. Unless otherwise specified, it is 
recommended to use quartz slides in order to eliminate as much as possible the influence of slide spectrum on the spectrum of the analyzed sample. Other substrates like a calcium fluoride are also available. Calcium fluoride could be used in the broad lasers' wavelength, with only on peak at around $320 \mathrm{~cm}^{-1}$. Also, in the recent years, SERS substrate based primarily on the silver nanoparticles are available, enabling Raman analysis of diluted samples.

If possible, one should avoid the use of plastic containers for holding samples during analysis because signals arising from the sample and from the container may overlap. In this case as well as when quartz plates are used, it is recommended to record spectrum of empty container prior to analysis, in order to avoid problems due to spectra overlapping.

\subsection{Analysis of solid samples}

Before the analysis it is necessary to properly apply a sample on the appropriate holder for recording. It is best to use quartz slides as this ensures minimum impact of the holder on Raman spectrum of the sample. When doing inverse Raman microscopy, the sample is analyzed from the bottom side and laser excitation of the sample is carried out from below. With such a configuration it is recommended to use a strip of two-sided-selfadhesive tape, first placing it onto a glass microscopic slide and then placing the sample on the other surface of the selfadhesive tape. Attention should be payed to possible negative impact of the strip material especially in the case of analyzing particles of smaller size and transparent samples because signals originating from the strip might appear in the sample spectrum.

\subsection{The analysis of liquid samples}

Most of the fluid samples may be analyzed immediately after application to the surface of the slide. This mode of recording is applicable for liquid samples that do not vary significantly (e.g. minimally evaporate) during analysis.

\section{The effect of laser wavelength on the quality of Raman spectrum}

The selection of the appropriate laser wavelength is certainly one of the most important tasks before starting the analysis (Figure 4).

When device is equipped with multiple lasers it is an easy procedure to change from one to the other laser. Before choosing the laser wavelength one should consult the literature from the field of investigation but also examine which laser is appropriate for particular sample. 


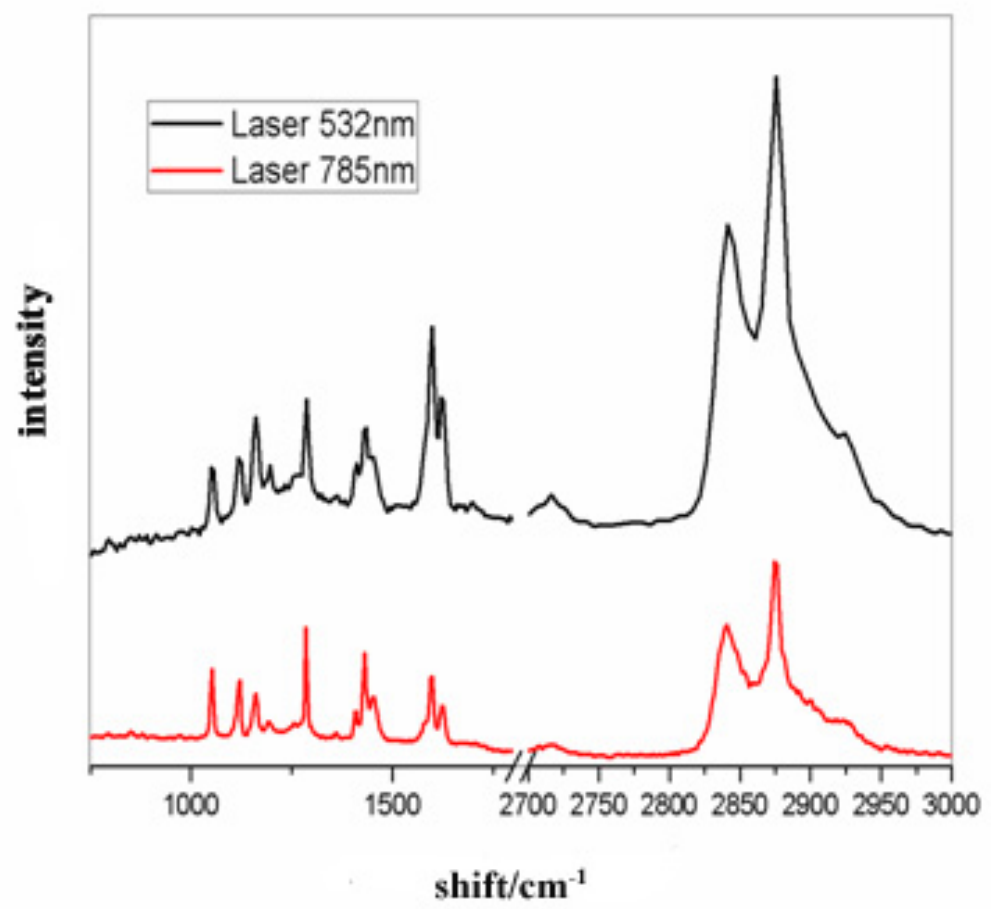

Figure 4: The effect of laser wavelength on Raman spectrum of carnauba wax.

However, the selection of the appropriate laser is limited to the available lasers that are installed. Lasers are part of larger optical system that includes adequate lenses, filters, spectrometer and detector.

\section{The impact of laser power on the quality of Raman signal}

The quality of the Raman spectra depends on the power of the applied laser. A general recommendation is to tend to obtain the maximum possible signal intensity. On the other hand it is necessary to take into account the stability of the samples that were exposed to the laser. In the Raman microscopy the laser is focused on a relatively small surface area which may lead to sample damage and can result in a signal which does not correspond to the actual spectrum of the sample. In extreme cases, too high intensity of the laser may completely disable the analysis due to the large sample damage. An example of such laser mediated damage of the sample is given in Figure 5.

It can be noticed that the laser greatly damaged the sample and therefore the analysis in these conditions is impossible. In order to prevent such a negative impact on the sample, the recommendation is to reduce the intensity of the 

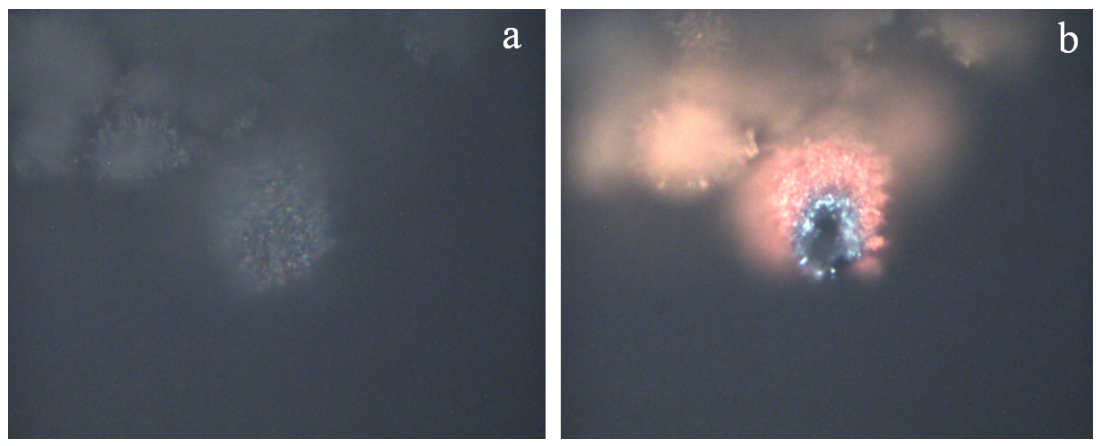

Figure 5: The impact of laser power on the sample: a) magnetite sample prior to analysis; b) after analyzing the sample of magnetite. Laser $532 \mathrm{~nm}$, filter $100 \%$, acquisition time of $1 \mathrm{~s}$, the lens $50 x L W D$, laser power $25 \mathrm{~mW}$.

laser in combination with shortening duration of acquisition. The $25 \mathrm{~mW}$ is enough, in the case of magnetite, to make considerable sample damage, while gradually reduction of laser power below $1 \mathrm{~mW}$ (and combined with adequate acquisition time) usually provides satisfactory results. Other materials, like a previously mentioned titanium dioxide or carnauba wax could be analyzed using even greater laser power (e.g. $125 \mathrm{~mW}$ ), without visible sample damage.

Note: it is recommended to inspect the surface of the sample after the analysis in order to determine whether there has been any damage. In many cases it is possible to notice during recording that sample has been damaged. If severe damage of the sample occurs while recording in real time, the spectrum is rapidly shifting and usually at some point signal disappears (spectrum completely lost). This is the sign to stop recording, inspect the sample, confirm the damage and then change the parameters of the analysis in order to obtain a spectrum of adequate quality and avoid the sample damage. However, as previously mentioned, Raman microscopy analysis should aim at getting signal of high intensity in order to interpret the results correctly.

The impact of the laser intensity on Raman spectrum quality is shown in Fig. 6.

Displayed spectra of titanium dioxide indicate that with any of the filters spectrum can be obtained, whereby the best signal is obtained at maximum intensity of the laser (filter 100\%). Here also the rule applies that the use of laser at maximal intensity is dependent of the stability of the analyzed sample.

\section{The effect of grooves density in the grating of spectrometer on quality of Raman signal}

The number of lines in the grating of the spectrometer is set before recording. It is recommended to start with a smaller number of grooves and then if the analysis requires it increase this number. Higher number of grooves assures 


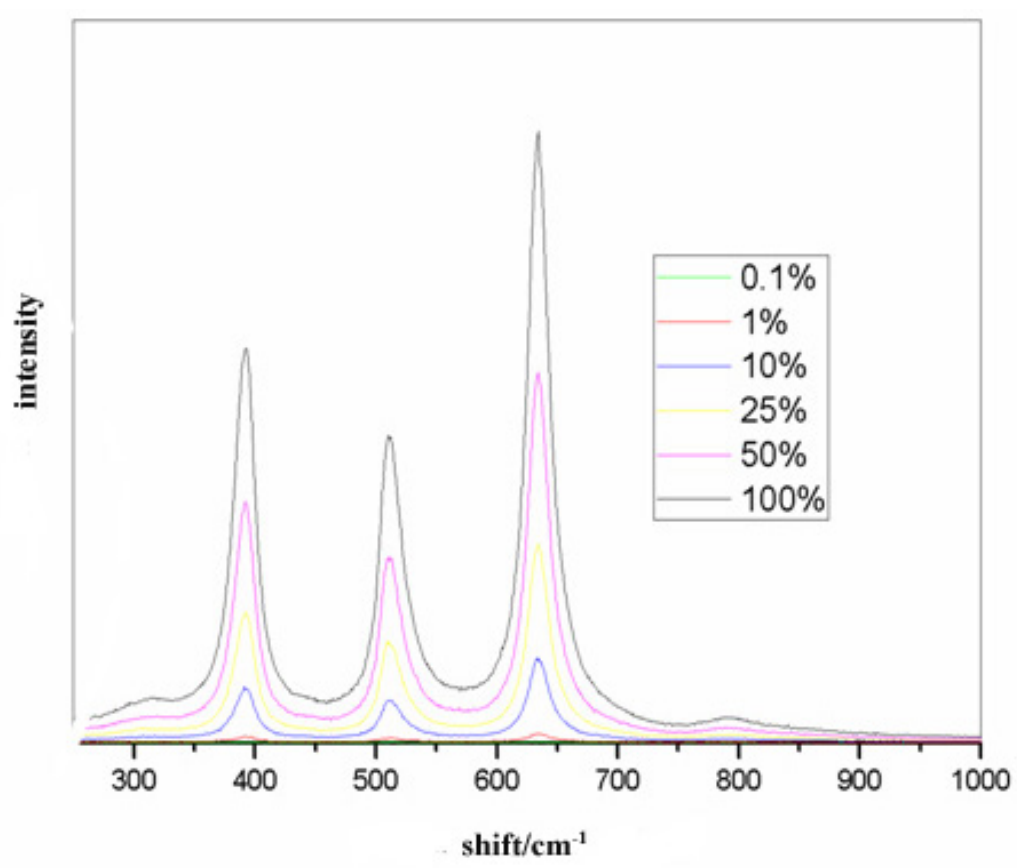

Figure 6: Effect of laser intensity on the quality of Raman spectrum of titanium dioxide. $532 \mathrm{~nm}$ laser, acquisition time of $1 \mathrm{~s}$, variable filters $0.1 \%, 1 \%, 10 \%$, $25 \%, 50 \%$ and $100 \%$.

better resolution and better separation of the peaks in the spectrum. It should be noted that using a higher number of grooves leads to the decrease in signal intensity as well as the increase in duration of recording. Signal intensity can be increased by increasing the duration of the recording.

Note: when recording involves a change in number of grating grooves, be sure to wait for the device to complete the procedure of changing the recording conditions to continue analysis under the new conditions. Program LabSpec 6 does not allow recording until the change of grooves number in the grating is not completed.

\section{The influence of analysis duration on the quality of Raman spectrum}

Duration of recording directly affects quality of the Raman signal. Generally, the longer is acquisition the obtained spectrum is more intense and therefore more reliable in terms of interpreting the results. Usually, the analysis of the 
sample begins with a shorter acquisition time which can then be extended in order to obtain a quality spectrum. On the other hand, too short acquisition time can lead to a drastic reduction in signal intensity and finally to a bad interpretation of the results.

Note: When selecting the time of acquisition one should pay attention to all possible phenomena. The first one is possible damage to the sample due to the long exposure to a laser. Another possible phenomenon is that the signal intensity is higher than the selected range of intensities, and the resulting spectrum is unusable. Therefore, the timing of acquisition is performed gradually, starting with a minimum of time that provides a signal and gradually increasing it until a maximal intensity of the spectrum is obtained. 
